\title{
Analysis of Disruptive Discharges During Density Limit Experiments on the HT-7 Tokamak
}

\author{
M. Asif, X. Gao, and the HT-7 Team \\ Institute of Plasma Physics, Chinese Academy of Sciences, P.O.Box 1126, Hefei, Anhui 230031, P.R. China
}

Received on 28 September, 2005

\begin{abstract}
In this work, we discuss the preliminary analysis of some disruptive plasma discharges with a moveable graphite limiter on the HT-7 tokamak, which has been developed in order to investigate the influence of the horizontal plasma position on the density limit. What we have observed is that the $m=2$, MHD component dominates during almost the whole discharge duration, when the density limit is approached at values of the edge safety factor $3<\mathrm{q}(\mathrm{a})<3.5$. For the poloidal-magnetic-field fluctuations observed at the Mirnov coils, Mirnov-coil analysis shows that the $\mathrm{m}=2$ mode is unstable, which finally causes disruption.
\end{abstract}

Keywords: Moveable limiter; Magneto-hydrodynamic; Mirnov coil; Disruptions

\section{INTRODUCTION}

Experimental results on TEXTOR have shown that the development of a MARFE (multifaceted asymmetric radiation from the edge), can be suppressed by only small changes in plasma position [1]. Similar results were obtained on TFTR [2] and on HT-7 [3]. Recently, a moveable graphite limiter [3], has been developed in order to investigate the influence of the horizontal plasma position on the density limit in the HT-7 tokamak. The purpose of this work is to optimize the configuration for synergy of high power LHW and IBW heating at high density in the HT-7 long pulse discharges [4]. Further investigations relating to this effect were not possible, due to magneto-hydrodynamic (MHD) instabilities have been observed when the density limit is approached at values of the edge safety factor $3<q(a)<3.5$, with a moveable graphite limiter [3], on the HT-7 tokamak. Here, the usual definition of $\mathrm{q}(\mathrm{a})$ is employed, i.e. $\mathrm{q}(\mathrm{a})=(\mathrm{a} / \mathrm{R})(\mathrm{B} \phi / \mathrm{Bp})$, where $\mathrm{Bp}=$ $\mu_{0} \mathrm{Ip} /(2 \pi \mathrm{a})$. Tearing modes are known to play an important role in confinement degradation and disruptions in tokamaks [5-6]. They are resistive instabilities driven by the free energy contained in the poloidal magnetic field. Due to their resonant character, they are localized around flux surfaces and change the topology of the magnetic-flux distribution through the formation of magnetic islands. At present, there exist several distinct MHD models of tokamak disruptions [7-10]. Understanding in detail the various mechanisms that can lead to disruption is important for achieving tokamak confinement, and especially so with a view to suppressing disruptions [11].

\section{EXPERIMENTAL SET-UP AND DIAGNOSTICS}

The Hefei tokamak-7 (HT-7) is a circular cross section superconducting tokamak [12-14], reconstructed from the original Russian T-7 tokamak in 1994. It has a major radius $\mathrm{R}$ $=1.22 \mathrm{~m}$, minor radius $\mathrm{a}=0.27 \mathrm{~m}$, defined by one poloidal water-cooling limiter, one toroidal water-cooling belt limiter at high field side and a modified set of actively cooled toroidal double-ring graphite limiters at bottom, and top of the vacuum vessel. Up to $240 \mathrm{~s}$ of plasma duration [4] has been achieved with new graphite limiters in the HT-7 in 2004. There are two layers of thick copper shells, and between them are located 24 superconducting coils which can create and maintain a toroidal magnetic field $\left(B_{T}\right)$ of up to $2.5 \mathrm{~T}$. The HT-7 ohmic heating transformer has an iron core and it can offer a magnetic flux of $1.7 \mathrm{Vs}$ at its maximum. In current experiments the position control was performed magnetically and the plasma column was limited by inserting the movable limiter [3], as shown in Fig. 1, to a minor radius of $a=0.25 \mathrm{~m}$. In this case it was possible to change the horizontal plasma position as shown in Fig. 2. The HT-7 tokamak is normally operated with $I_{P}=100-250 \mathrm{kA}, B_{T}=1 \sim 2.5 \mathrm{~T}$, line-averaged density $1-6 \times 10^{19} \mathrm{~m}^{-3}$.

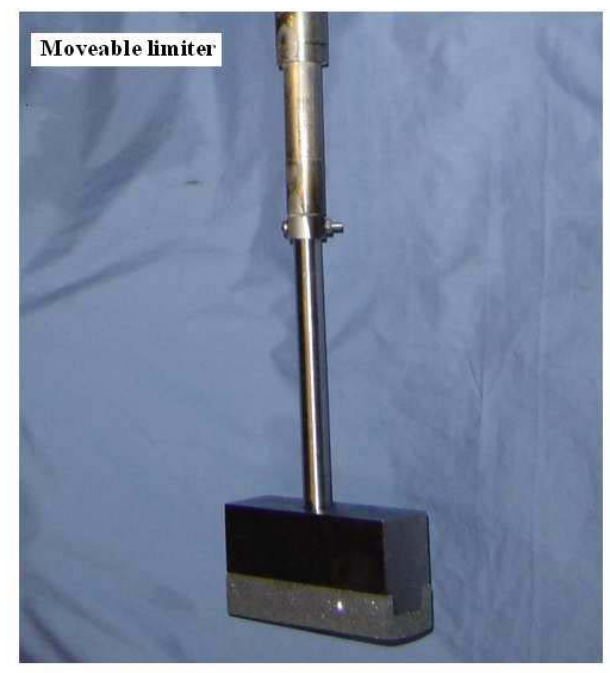

FIG. 1: A photo of moveable limiter with graphite tip.

The main diagnostics for recent experiments on the HT7 are the electron density profile measured by a vertical 5channel far-infrared (FIR) hydrogen cyanide (HCN) laser interferometer, a multi-channel soft x-ray array, an electron cyclotron emission diagnostic, Soft x-ray pulse-height-analysis (SX-PHA) diagnostic, a 16-channel XUV bolometer array 


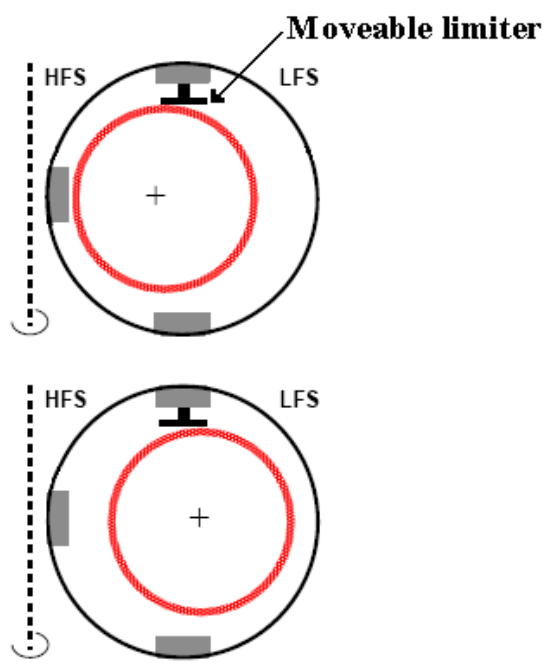

FIG. 2: Two schematic views of the plasma poloidal cross section for outwards (left) and inwards (right) shifted discharge. The plasma boundary is determined by the moveable limiter.

to measure plasma radiation losses, a multichannel $\mathrm{H}_{\alpha}\left(\mathrm{D}_{\alpha}\right)$ radiation array, 4-channel visible bremsstrahlung emission, 10-channels CIII line emission, an impurity optical spectrum measurement system, and a magnetic measurement system for plasma current, loop voltage, plasma position, internal inductance and so on.

The magnetic fluctuations are observed with two arrays of magnetic probes which are set up on the inside of the vacuum vessel wall at different positions in the toroidal direction. Each array has 12 probes around the plasma in a circular section. From the outer-middle plane, every probe is installed at $30^{\circ}$ with respect to the nearest neighbouring probes. The frequency response of the probe is more than $100 \mathrm{kHz}$. The effective winding area is $1430 \mathrm{~cm}^{2}$. Of particular importance is the detection of specific modes from the Mirnov-coil signals. Upon time-integrating a Mirnov signal we find the perturbed poloidal field, from which a given mode can be singled out by means of Mirnov-coil analysis. The spatial structure and temporal evolution of coherent MHD modes observed in the HT-7 superconducting tokamak have been inferred from the experimental signals by using spectral techniques. Using multichannel fluctuation data analysis by the singular value decomposition method (SVD) [15-16], the poloidal number $\mathrm{m}$ is obtained. Fig. 3 shows a typical shot where large MHD activity leads to a disruption, when the density limit is approached at values of the edge safety factor $3<q(a)<3.5$.

\section{RESULTS AND DISCUSSION}

In order to investigate the influence of the plasma position on the density limit, we reduced the plasma minor radius to

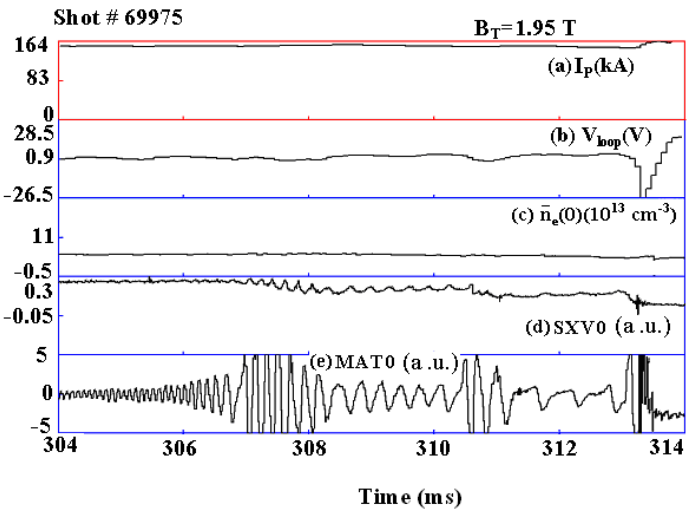

FIG. 3: Typical HT-7 discharge with MHD disruption. (a) plasma current, (b) loop voltage, (c) central line-averaged density, (d) central chord soft x-ray emission SXV0, (e) Mirnov-coil signal MAT0, when the plasma column was repositioned more than $\Delta_{/ /}=+2.50 \mathrm{~cm}$ outwards.

$a=25 \mathrm{~cm}$ by inserting a moveable graphite limiter. The horizontal plasma position was then changed from discharge to discharge. The plasma position control signal was generated by magnetic diagnostics. For these ohmic discharge series the toroidal plasma current was $\mathrm{I}_{P} \sim 154 \mathrm{kA}$, which refers to a Greenwald limit [17], of $n^{G W} \sim 7.8 \times 10^{19} \mathrm{~m}^{-3}$. The density was increased by normal gas puffing, up to a disruption. Fig. 3 shows the typical temporal evolution of the MHD behavior, obtained in shot of experimental campaign 2004 (shot No.69975) on HT-7, the plasma current about $154 \mathrm{kA}$, the loop voltage $\mathrm{V}_{\text {loop }}<3 \mathrm{~V}$, the toroidal field $\mathrm{B}_{T}=1.95 \mathrm{~T}, \mathrm{R}=1.22$ $\mathrm{m}, \mathrm{a}=0.25 \mathrm{~m}$ and the line-averaged density $\mathrm{n}_{e}=7 \times 10^{19} \mathrm{~m}^{-3}$.

It has been observed when the density reaches $90 \%$ of the predicted Greenwald limit, a large MHD activity which leads to the disruption, as the plasma column was repositioned more than $\Delta_{/ /}=+2.50 \mathrm{~cm}$ outwards. The effective charge $\mathrm{Z}_{\text {eff }}$ measured by visible bremsstrahlung emission intensity in these discharges was quite similar, $\mathrm{Z}_{e f f} \cong 1.25$. A development of the event preceding the disruption has been observed for different position settings. The shifted discharges, located near the HFS wall and the LFS wall (see Fig. 3), showed clear indication of the appearance of a MHD activity.

Magnetic analysis by singular value decomposition method (SVD) [15-16] for the discharge in Fig. 3 shows that the MHD activity consists predominantly of $m=2$ modes with a rotation frequency of about $7.8 \mathrm{kHz}$, the major disruption was triggered at $314.5 \mathrm{~ms}$ (see Fig. 4).

In HT-7, the most dangerous MHD instability is the $\mathrm{m} / \mathrm{n}$ $=2 / 1$ resistive tearing mode, which is driven by the plasma current density gradient. In this experiment, at $q(a)=3.2$, no mode locking is observed, the observed MHD activity can be associated with tearing mode, a non-linear instability with low $\mathrm{m} / \mathrm{n}$ modes. The physical mechanism, however, which finally triggers the MHD disruption in high $\mathrm{q}(\mathrm{a})(>3)$ deuterium plasmas is essentially the same [5]. The poloidally asymmetric increase of the plasma resistivity due to MARFE cooling 
(a) chrono
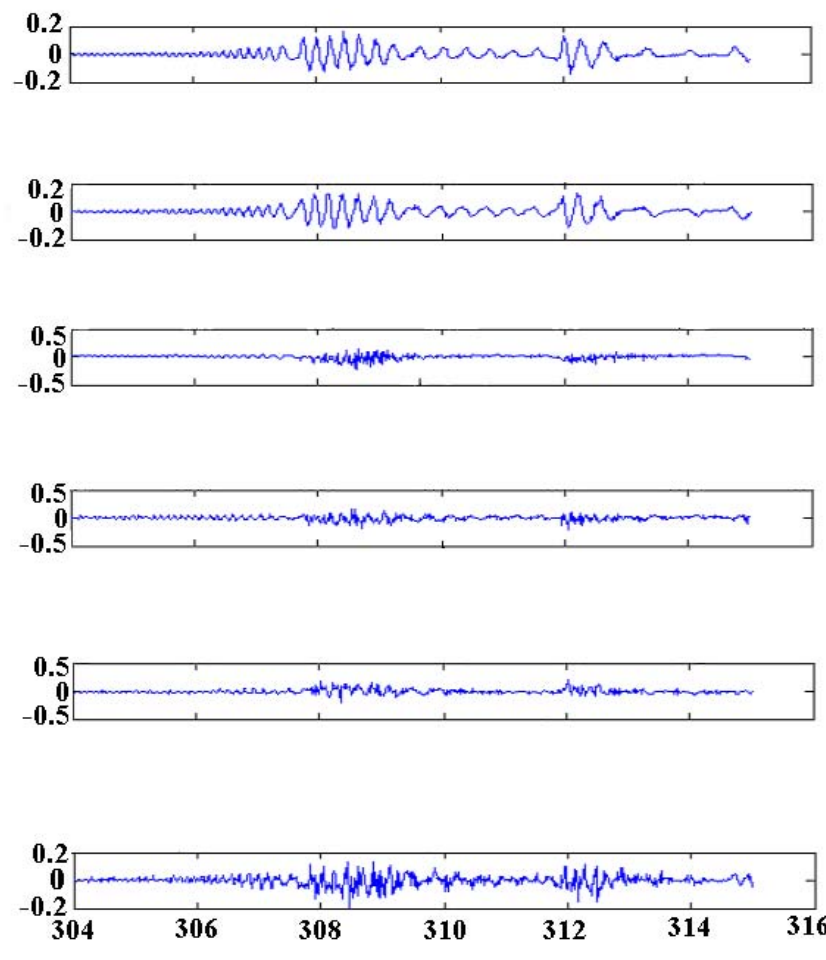

Time (ms) (b) topo
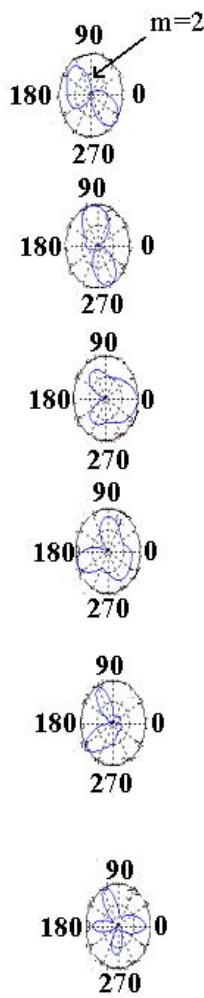

FIG. 4: Results of the SVD analysis from magnetic signals. (a) the first order temporal eigenvector of the mode, (b) the spatial eigenvector corresponding to the temporal eigenvector in Fig. 2(a). The main mode number is $m=2$.

leads to a global contraction of the current channel, as has been shown theoretically by Lackner [18]. Qualitatively, because of the equilibrium constraint, the Ohmic resistance of cold MARFE (a few eV) appears in series with the hot plasma resistance (several tens of $\mathrm{eV}$ ) on each flux surface, resulting in a substantial increase of the average edge resistivity. As a consequence of the current contraction, the gradient of $j(r)$ is steepened, leading to a higher value of the instability parameter $\Delta^{\prime}$. This results in the formation of quasi-stationary, rotating islands, located near the rational surface, for $m / n=2 / 1$ mode the $q=2$ surface. After knowing the current density profile, $J \sim\left(1-X^{2}\right)^{\alpha}$, where $\mathrm{X}=\mathrm{r} / \mathrm{a}$, the q-profile can be calculated by using the formula

$$
q(r)=q(a)^{X^{2}} /\left[1-\left(1-X^{2}\right)^{\alpha+1}\right] .
$$

For shot no. $69975, q(a)=3.2$, and $\alpha=3$, the corresponding q-profile is

$$
q(r)=3.2^{X^{2}} /\left[1-\left(1-X^{2}\right)^{4}\right] .
$$

Figure 5 shows the calculated $q(r)$ profile (shot no. 69975). In the range of the MHD activity, $m=2$ modes, the resonant layer of $q$ is located at $0.7 a$ as shown in Fig. 5.

\section{CONCLUSIONS}

In this work we have described the cause of disrupting deutrium plasmas in the ohmic-heating regime on the HT7 tokamak in order to investigate the influence of the horizontal plasma position on the density limit. The purpose of this work is to optimize the configuration for synergy of high power LHW and IBW heating at high density in the HT-7 long pulse discharges. In recent experiments, unfortunately, further 


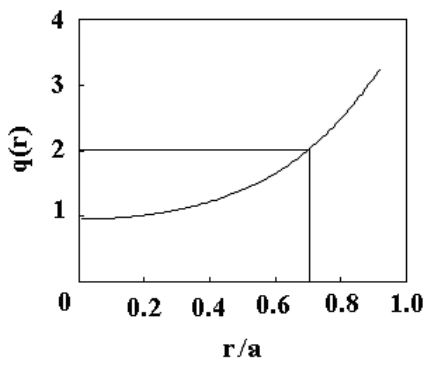

FIG. 5: Calculated q(r) profile for discharge No. 69975. investigations relating to this effect were not possible, due to the magneto-hydrodynamic (MHD) instabilities that have been observed when the density limit is approached at values of the edge safety factor $3<\mathrm{q}(\mathrm{a})<3.5$, with a moveable graphite limiter, on the HT-7 tokamak.

For the poloidal-magnetic-field fluctuations observed at the Mirnov coils, Mirnov-coil analysis shows that the $\mathrm{m}=2$ mode is unstable. Moreover, we do not know some of the detailed parameter profiles, such as $\mathrm{q}(\mathrm{r})$. We propose that the mode may be destabilized by the current density gradient.

\section{Acknowledgements}

The authors wish to thank the HT-7 team for cooperation during these experiments.
[1] U. Samm, et al., in Controlled Fusion and Plasma Physics (Proc. 18th Int. Conf. Berlin, 1991), 15C, Part III, 137 (1991) .

[2] J. F. Schivell, et al., Fusion Technol. 15, 1520 (1989).

[3] M. Asif, et al., Phys. Lett. A. 336, 61 (2005).

[4] M. Asif, et al., Phys. Plasmas 12, 082502 (2005).

[5] J. A. Wesson, R. D. Gill, M. Hugon, et al. Nucl. Fusion 29, 641 (1989).

[6] F. C. Schuller, Plasma Phys. Control. Fusion 37, A135 (1995).

[7] A. Sykes and J. A. Wesson, Phys. Rev. Lett. 44, 1215 (1980).

[8] H. R. Hicks, B. A. Carreras, J. A. Holmes, and V. E. Lynch, Nucl. Fusion 22, 117 (1982).

[9] M. F. Turner and J. A. Wesson, Nucl. Fusion 22, 1069 (1982).
[10] R. B. White, D. A. Monticello, and M. N. Rosenbluth, Phys. Rev. Lett. 39, 1618 (1977).

[11] A. Vannucci, R. D. Gill, Nucl. Fusion 31, 1127 (1991).

[12] M. Asif, et al., Phys. Lett. A. 342, 175 (2005).

[13] M. Asif, et al., Phys. Lett. A. 346, 305 (2005).

[14] M. Asif, et al., Phys. Lett. A. 349, 250 (2006).

[15] C. Nardone, Plasma Phys. Control. Fusion 34, 1447 (1992).

[16] T. Dudok de Wit, A.L. Pecquet, and J.C. Vallet, Phys. Plasmas 1, 2288 (1994).

[17] M. Greenwald, et al., Nucl. Fusion 28, 2199 (1988).

[18] K. Lackner (IPP Garching), personal communication,1991. 